\title{
An interpreter advantage in executive functions?
}

\section{A systematic review}

\section{Soudabeh Nour $^{1,2}$, Esli Struys ${ }^{1,2,3,4}$, Evy Woumans ${ }^{5}$, Ily Hollebeke ${ }^{2}$, \& Hélène Stengers ${ }^{2}$}

${ }^{1}$ Brussels Institute for Applied Linguistics, Vrije Universiteit Brussel

${ }^{2}$ Centre for Neurosciences, Vrije Universiteit Brussel, Belgium

${ }^{3}$ Centre for Linguistics, Vrije Universiteit Brussel, Belgium

${ }^{4}$ Leiden University Centre for Linguistics, Leiden University, The Netherlands

${ }^{5}$ Department of Experimental Psychology, Ghent University, Belgium 
Soudabeh Nour

Pleinlaan 5 -1050 Brussel, Belgium

Soudabeh.Nour@vub.ac.be

Esli Struys

Pleinlaan 2 -1050 Brussel, Belgium

estruys@vub.ac.be

Evy Woumans

Henri Dunantlaan 2 - 9000 Gent, Belgium

Evy.Woumans@UGent.be

Ily Hollebeke

Pleinlaan 5 - 1050 Brussel, Belgium

ily.hollebeke@gmail.com

Helene Stengers

Pleinlaan 5 - 1050 Brussel, Belgium

Helene.Stengers@vub.ac.be 


\begin{abstract}
The aim of this systematic literature review was to answer the question of which type of executive functions is most affected by interpreter training and experience. We used the 'unity and diversity' framework of executive functions to distinguish between three executive components: Response and Distractor Inhibition, Shifting, and Updating. We classified seventeen previous studies according to which of these three executive functions had been measured. We only found evidence for an interpreter advantage on Shifting and Updating, but with a different pattern for each of these. With regard to Updating, groups of interpreters scored better than control groups, but longitudinal studies did not show an improvement for interpreter trainees. In contrast, for Shifting, scores improved as a result of interpreting training and experience alike. Our systematic review stresses the importance of understanding the diversity of executive processes when investigating the interaction between interpreting and cognitive performance.
\end{abstract}

\title{
Key words
}

Interpreting, cognitive control, executive functions, bilingual advantage, shifting 


\section{Biosketch Soudabeh Nour}

Soudabeh Nour is a PhD student in applied linguistics at the Brussels Institute for Applied Linguistics of the Vrije Universiteit Brussel. She is working on a project investigating the relation between cognitive control components and interpreting practice. Her research activities include the neurocognitive investigation of multilingualism and interpreting.

\section{Biosketch Esli Struys}

Esli Struys is an assistant professor of multilingualism and interpreting at the Department of Linguistics and Literary Studies of the Vrije Universiteit Brussel in Belgium. He teaches courses on multilingualism and interpreting studies in the Bachelor's programme of Applied Linguistics and the Master's programmes of Linguistics and Literary Studies, and Interpreting. His research activities include the neurocognitive investigation of multilingualism, interpreting and multilingual education.

\section{Biosketch Evy Woumans}

Evy Woumans obtained her PhD in Psychology at Ghent University in 2015 by investigating the effects of bilingualism on cognitive functioning. She is now a postdoc on the LEMMA project at the department of Experimental Psychology (Ghent University), conducting research on the interface between language and memory in multilingual education.

\section{Biosketch Ily Hollebeke}

Ily Hollebeke obtained a Master's degree in Interpreting at the Vrije Universiteit Brussel and is currently enrolled in a postgraduate programme in Conference Interpreting at Ghent University. She wrote her Master's thesis on cognitive abilities in interpreter trainees.

\section{Biosketch Helene Stengers}

Helene Stengers is professor in Spanish proficiency, Translation and Interpreting at the Vrije Universiteit Brussel, where she obtained her PhD in 2009. Her research interests lie in applied comparative linguistics (especially English and Spanish), cognitive linguistics, phraseology and Foreign Language acquisition (mainly vocabulary acquisition) from a multilingual and intercultural perspective with a view to optimize Foreign Language pedagogy, as well as translation and interpreter training. 


\section{Introduction}

Executive functions (or cognitive control) refer to a set of mental processes that regulate cognition, such as the ability to suppress task-irrelevant responses or to shift between mental sets (e.g. Diamond, 2013; Miyake et al., 2000). Language interpreting has drawn the attention of scholars interested in executive functions because it is a complex activity that requires the simultaneous activation of two mental language sets in two different modalities (e.g. Injoque-Ricle, Barreyro, Formoso, \& Jaichenco, 2015; Yudes, Macizo, \& Bajo, 2011). As many studies on the interaction between interpreting and executive functions show enhanced performance for groups of interpreters as compared to control groups (e.g. Christoffels, de Groot, \& Kroll, 2006), an interpreter advantage hypothesis has been proposed, which posits that constant practice or training in the domain-specific skills engaged in interpreting transfers into a domain-general cognitive advantage (Garcia, 2014).

Given that executive functions refer to a multitude of processes, the question arises whether interpreting has a general effect on these processes or only influences specific subcomponents. This question can be answered through the various cognitive subdomains in which the interpreter advantage has been studied, one example being working memory capacity (e.g. Cai, Dong, Zhao, \& Lin, 2015; Van Dijk, Christoffels, Postma, \& Hermans, 2012; Wang, 2016); other including task switching (or shifting) ability (e.g. Dong \& Liu, 2016; Dong \& Xie, 2014; Macnamara, Moore, Kegl, \& Conway, 2011), mental (or cognitive) flexibility (e.g. Macnamara et al., 2011; Yudes et al., 2011), inhibition (e.g. Babcock \& Vallesi, 2015; Dong \& Xie, 2014), conflict resolution (e.g. Babcock \& Vallesi, in pressin press), selective attention (e.g. Morales, Padilla, Gomez-Ariza, \& Bajo, 2015; Woumans, Ceuleers, Van der Linden, Szmalec, \& Duyck, 2015), updating (e.g. Dong \& Liu, 2016) and dual tasking (e.g. Strobach, Becker, Schubert, \& Kuhn, 2015).

These studies have revealed mixed results regarding the question of which of these subdomains are selectively influenced by interpreting (compare, for instance, Timarova et al., 2014 who show a selective advantage on inhibition but not on flexibility to Yudes et al., 2011 who show a selective advantage on cognitive flexibility but not on inhibition). We suggest that these conflicting results can be explained by understanding how the investigated cognitive subdomains relate to each other, as demonstrated by theoretical frameworks on working memory and executive functions, and by revealing how the interpreter advantage hypothesis has been tested in a variety of research designs. 
The field of interpreting and cognition has seen a major shift from an initially selective interest in (working) memory (e.g. Christoffels et al., 2006) to a more wider focus on executive functions (e.g. Timarova et al., 2014). The concept of working memory is to a large extent related to that of executive functions, and some scholars have even conceptualised the latter as a subset of the mental processes involved in working memory (e.g. Baddeley, 1992). According to one influential model (Hitch \& Baddeley, 1976), working memory can be subdivided into a central executive responsible for the manipulation or processing of information, and two slave systems that store information: the phonological loop and the visuo-spatial sketch pad. However, not all experts of working memory share the idea that it is composed of storage and processing components. An alternative view states that working memory only refers to the processing of information, and thus to the central executive, while the storage component is referred to as short-term memory capacity (e.g. Colom, Rebollo, Abad, \& Shih, 2006; Unsworth \& Engle, 2006, 2007). Despite the obvious differences between these two viewpoints, they converge on the idea that the (central) executive functions are involved with the manipulation of information, and not with its storage. However, they reveal little about the variety of executive functions.

A coherent framework on how various cognitive control subdomains relate to each other is provided by the influential 'unity and diversity' model of executive functions (Miyake et al., 2000). This model has some clear advantages that may help us to specify in which subdomains interpreter advantages of executive functioning can be found. A first advantage is that this model has been empirically validated by latent variable analyses on scores from nine widely used tasks of executive functioning. These latent variables were labeled 'inhibition of prepotent responses' (or, Inhibition), 'shifting between mental sets' (or, Shifting), and 'updating of working memory representations' (or, Updating), each of which could be related to scores on three of the nine examined tasks. A second advantage of the 'unity and diversity' model is that structural equation modelling was used to determine how these three latent variables contribute to five additional complex tasks of executive functioning. For four of these tasks, a relationship could be established with one of the three latent variables (i.e. Inhibition, Shifting, and Updating). In sum, the 'unity and diversity' model allows for a consistent classification of eleven of the most widely used tasks of executive functions into three subdomains and this classification can be used for a valid comparison of executive functioning across studies. 
Not only the executive processes being tested vary across studies, the literature also shows a wide range of research designs that have been used to test the interpreter advantage hypothesis. In its most straightforward form, the interpreter advantage refers to the finding of superior performance for groups of interpreters compared to control groups of matched noninterpreters being tested cross-sectionally or at a single point in time (e.g. Christoffels et al., 2006; Morales et al., 2015; Yudes et al., 2011). In addition to this pure interpreter advantage, the hypothesis of an interpreter advantage has also been tested and confirmed by revealing enhanced executive functioning for interpreters with professional experience compared to non-experienced interpreters (Köpke \& Nespoulous, 2006; Liu, Schallert, \& Carroll, 2004); for interpreters with more years of training compared to interpreters at the initial stages of their training (Dong \& Xie, 2014; Tzou, Eslami, Chen, \& Vaid, 2012); and for well-skilled interpreters compared to low-skilled interpreters (Macnamara et al., 2011), all using a crosssectional design.

Cross-sectional between-group analyses are characterised by some drawbacks with regards to the interpretation of the reported results. One major disadvantage of between-group analyses is that they neglect individual variability within the interpreting community or within an interpreting sample based on one aspect of the interpreting experience, such as the quality of interpreting. Possibly, these individual differences are related to specific and quantifiable aspects of the interpreting experience (e.g. the amount of professional experience, the amount of training, etc.) that are sensitive enough to show variation within the interpreting population. In line with this assumption, a few studies have revealed significant correlations between scores on executive functioning tasks, on the one hand, and number of years of interpreting experience (Timarova et al., 2014), measures of interpreting quality (Cai et al., 2015; InjoqueRicle et al., 2015; Wang, 2016).

Another disadvantage of cross-sectional studies is that no causal relationships can be inferred from them. The finding of superior performance for interpreters over non-interpreters in a cross-sectional design can be explained from two opposing perspectives. One option is that that the observed differences are related to pre-existing individual (genetic or environmental) differences (compare to Hernandez, Greene, Vaughn, Francis, \& Grigorenko, 2015) and that only individuals with high initial abilities are allowed (or encouraged) to enter training programmes of interpreting or are able to proceed to the interpreting profession. An alternative option is that interpreter training or prolonged practice itself changes the strength and efficiency of executive functions, irrespective of initial cognitive ability. Only 
longitudinal research designs with measurements before and after a specific intervention can disentangle both options and determine the directionality of the observed effects. The usage of this type of designs has revealed significant progress on executive functions after having received training in interpreting (Macnamara \& Conway, 2014), even when interpreter trainees are compared to students of language and culture or of translation (Dong \& Liu, 2016).

\section{The present study}

Based on the 'unity and diversity' model of executive functions and reflecting the variety of interpreter advantages that can be found in the literature on the interaction between interpreting and executive processes, this systematic review intends to answer three research questions:

1) The first question is on which executive process (Inhibition, Shifting, or Updating) interpreters score better than controls. This question can be answered by considering cross-sectional studies with between-group comparisons.

2) The second question is which executive process related to interpreting experience. This can be answered by an analysis of cross-sectional correlational or between-group studies.

3) The third question is which executive process shows most progress during interpreter training. This can be answered mainly by looking at longitudinal studies.

In line with recent neuroimaging experiments that have revealed a selective effect of interpreting performance and training on neural regions involved in executive functioning (Hervais-Adelman, Moser-Mercer, \& Golestani, 2015; Hervais-Adelman, Moser-Mercer, Michel, \& Golestani, 2015), we expect interpreting to have an effect on at least one of the three considered types of executive functions. Moreover, we assume that the distinction between the first and the two other research questions may be helpful in drawing conclusions on the causal relationship between interpreting and executive functions. Potential differences in response to research questions one, two, and three may reveal which executive processes are important in the selection process of interpreter trainees or professional interpreters, and which executive processes change as a result of interpreting experience and training. As such, this review could shed light on the malleability of each of the three considered executive processes as a function of intense language training. 


\section{Methods}

\section{Information sources and search}

Three databases (Google Scholar, ScienceDirect, Web of Science) were searched with a cut-off date of December $1^{\text {st }}, 2016$. All of the searches used variations of the following terms and key words: interpret(er)(ing), executive function(s)(ing) or process(es)(ing), cognitive control, shift(ing), update(e)(ing), inhibit(ion), and (working) memory. Tables of contents were inspected in peer-reviewed journals that focus on translation, interpreting and bilingualism-related topics. The electronic search, conducted by two authors independently, scanned each title and abstract, retrieving articles on the basis of their relevance to interpreting and cognitive control. Along with review papers, some authors also were contacted through ResearchGate for additional information about their articles. Furthermore, the reference lists of publications located through these search methods were also inspected to identify studies cited therein. The inclusion criteria listed below were applied to the respective shortlists of papers.

\section{Eligibility criteria and study selection}

A set of inclusion criteria was defined to identify whether the considered articles were fitted for this review. The set of inclusion criteria are as follow:

1) The original research article was published in a peer-reviewed scientific journal.

2) The study population included at least one sample of 'professional interpreters' or 'interpreter trainees'.

3) The materials used in the study included at least one task of executive functioning. Task inclusion was rather based on the task characteristics than on the label given by the authors.

4) Language of the study was English.

5) The study reported statistical analysis of the collected data.

\section{Data collection process}

Classification according to research design

A first distinction was made between studies that tested the participants at one single point in time (cross-sectional studies) and studies that tested the participants at two or more 
points in time (longitudinal studies). A second distinction was made between studies that compared performance of at least two groups (between-group comparisons), one of which was a group of interpreters, and studies that used an individual differences approach and investigated the relationship between at least one measure of executive functioning and one measure of interpreting experience (correlational analyses). The first research question of the

present study was answered by systematically reviewing cross-sectional studies with betweengroup comparisons. Cross-sectional studies with correlational analyses were systematically reviewed to answer the second research question; finally, a review of longitudinal studies was the basis for answering the third research question of this systematic review. It should be noted that cross-sectional studies with between-group comparisons that involved more than one group of interpreters based on interpreter experience or the length of interpreter training could also be used to answer the second and third research questions, respectively.

\section{Classification according to task of executive functioning}

These tasks were classified into one of the three executive processes included in the 'unity and diversity' model (Miyake et al., 2000): Inhibition, Shifting, and Updating.

First, Inhibition refers to one's ability to deliberately inhibit dominant, automatic or prepotent responses when necessary. In the present study, a task was automatically classified into this component if it was one of the tasks mentioned in the 'unity and diversity' model (Miyake et al., 2000) as valid measures of Inhibition: the antisaccade task (Hallett, 1978), the stop-signal task (Logan, 1994), the Stroop task (Stroop, 1935), or the Tower of Hanoi (Arnett et al., 1997); or if it was one of the tasks that taps into 'Resistance to Distractor Interference', as measured, among others, by the flanker task or Simon task (Eriksen \& Eriksen, 1974; for more information about the relation between Inhibition and Resistance to Distractor Interference, see Friedman \& Miyake, 2004; Simon \& Rudell, 1967). For clarity's sake we chose to update the label 'Inhibition' to 'Response-Distractor Inhibition' (Friedman \& Miyake, 2004). Measures of 'Response-Distractor Inhibition' can also be embedded within tests of attentional networks, such as the Attention Network Test (ANT; Fan, McCandliss, Sommer, Raz, \& Posner, 2002). The ANT is a combination of a flanker task and a cuing task (Posner, 1980) and taps into the attentional networks of alerting, orienting, and executive functioning. Only the ANT measures of executive functioning, which are in essence flanker trials, were included in this review. 
Second, Shifting is concerned with shifting back and forth between multiple tasks, operation, or mental sets (Monsell, 1996). In the present study, a task was automatically classified into this component if it was one of the tasks mentioned in the 'unity and diversity' model (Miyake et al., 2000) as valid measures of Shifting: any task-switching paradigm such as the plus-minus task (Jersild, 1927), the number and letter task (Rogers \& Monsell, 1995), the local-global task (Navon, 1977), or the Wisconsin Card Sorting Test (WCST; Berg, 1948). Based on its described characteristics, a task could also be classified into the Shifting component if it met the definition given by the 'unity and diversity' model (Miyake et al., 2000). It should be noted that task switching paradigms do not only tap into shifting abilities, but may also elicit the recruitment of inhibitory processes (for a review, see Koch, Gade, Schuch, \& Philipp, 2010). This is especially so for the $n-2$ repetition cost (e.g. Scheil \& Kleinsorge, 2014), which refers to the additional time that is needed to access a task that was presented two trials before the current trial, but was inhibited on the previous trial. As most research seems to converge on the idea that this n-2 repetition cost in task-switching paradigms reflects the recruitment of inhibitory processes (e.g. Koch, Gade, \& Philipp, 2004; Philipp, Gade, \& Koch, 2007; Philipp \& Koch, 2009), this cost was included as a measure of 'Response-Distractor Inhibition' instead of 'Shifting'.

Third, Updating refers to the cognitive processes needed for monitoring and coding incoming information for its relevance to the task and appropriately revising and replacing old with new information in working memory (Morris \& Jones, 1990). In the present study, a task was automatically classified into this component if it was one of the tasks mentioned in the 'unity and diversity' model (Miyake et al., 2000) as valid measures of Updating: the keep track task (Yntema, 1963), the letter memory task (Morris \& Jones, 1990), the $n$-back task (Jonides \& Smith, 1997), the tone monitoring task (Larson, Merritt, \& Williams, 1988), and complex working memory span tasks (Bayliss, Jarrold, Gunn, \& Baddeley, 2003). We chose not to include simple working memory span tasks because they are assumed to be related more to the storage capacity of short-term memory than to working memory updating abilities (Unsworth \& Engle, 2007).

Furthermore, we did not incorporate tasks that either are not related to any of the three executive processes as identified by the 'unity and diversity' model (Miyake et al., 2000), such as dual-tasking, or that are measures of Resistance of Proactive Interference, which is a construct unrelated to Response-Distractor Inhibition (Friedman \& Miyake, 2004), as well as the studies which did not answer any of three main research questions. 


\section{The results of the administered tasks}

To answer the first and third research questions (and in some occasions also the second research question), either $F$ - or $t$-values were extracted from cross-sectional studies with between-group comparisons or from longitudinal studies. Main effects of group or time, or an interaction effect between these two factors as indicated by $p$-values below .05 were treated as indicators of positive answers to the first (and, in a few instances, also to the second) and third research questions, respectively. To answer the second research question, in nearly all instances, $r$-values were extracted from cross-sectional studies with correlational analyses. A significant correlation as indicated by a $p$-value below .05 was treated as an indicator of a positive answer to the second research question.

\section{Results}

\section{Data selection}

The electronic search resulted in a total of 1,798 articles. Many articles were included in more than one database; therefore, duplicate articles were removed first. Then, two researchers reviewed the titles, abstracts, and keywords of these articles for possible inclusion by applying the selection criteria stated above. When abstracts did not contain sufficient information to determine inclusion or exclusion, the full text of the article was obtained and read. Articles that did not meet the selection criteria were excluded. In total, 17 papers met our strict inclusion criteria (see Figure 1). 


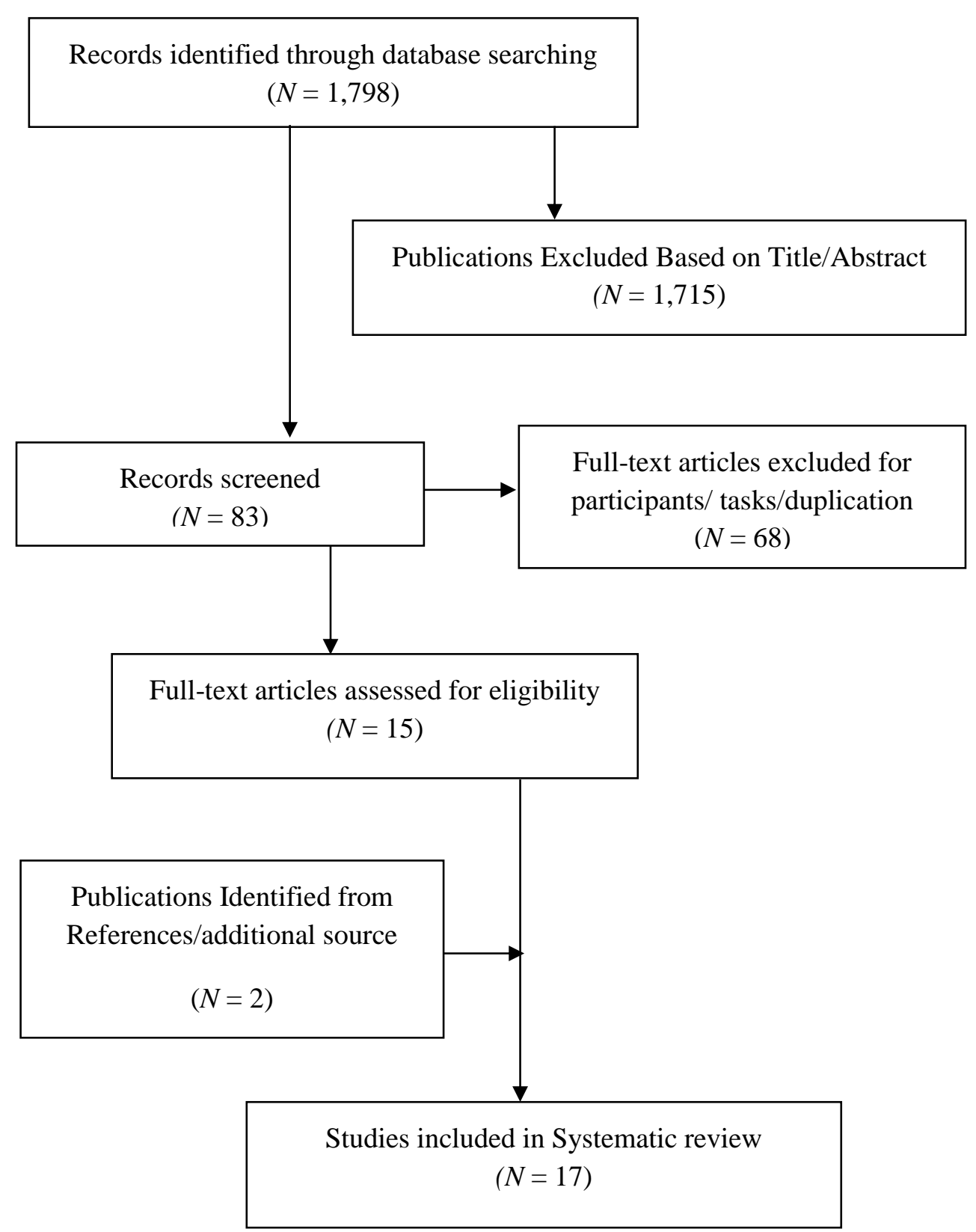

Figure 1. Flow diagram of articles selected for systematic review

\section{Research designs of the reviewed studies}

Information on the research designs that were used in these 17 studies on the interpreter advantage in executive functions is given in Table 1. 
Table 1. Overview of the research designs in studies on the interpreter advantage in executive functions. It should be noted that one study reports on experiments with cross-sectional and longitudinal designs, and is therefore counted twice in this overview.

\begin{tabular}{lll}
\hline \hline Number of time points & Grouping characteristics & Number of studies \\
\hline One: Cross-sectional design & Between-group comparisons & 7 \\
& with one group of interpreters & \\
\cline { 2 - 2 } & Between-group comparisons & 6 \\
& with more than one group of \\
& interpreters & \\
& & \\
\cline { 2 - 2 } & Correlational analyses & 2 \\
\hline Two or more: Longitudinal design & 3
\end{tabular}

\section{Administered tasks of executive functioning}

These 17 articles report on a total of 94 tasks with 1,176 participants. From these 94 tasks, only 38 tasks were included in this review because they could be classified into one of the three executive functioning (EF) components revealed by the 'unity and diversity' model (Friedman \& Miyake, 2004; Miyake et al., 2000): Response-Distractor Inhibition, Shifting and Updating as well is directly addressing at least one of there research questions of this review. An overview of the tasks included in this review, and the number of measures for each of the three EF components can be found in Table 2. 
Table 2. Overview of the tasks included and their number for each of the three EF components.

\begin{tabular}{llc}
\hline \hline EF component & Tasks included & Number \\
\hline $\begin{array}{l}\text { Response-Distractor } \\
\text { Inhibition }\end{array}$ & ANT (only executive functioning contrast), Anti-saccade, & 12 \\
& $\begin{array}{l}\text { Flanker, Simon, Stroop, Task switching paradigm (only n-2 } \\
\text { repetition cost) }\end{array}$ & \\
\hline Shifting & Number-letter, Task switching paradigm (only switch & 7 \\
& cost), WCST & 19 \\
\hline Updating & Complex span, $n$-back & \\
\hline \hline
\end{tabular}

\section{Results of the included studies}

Based on the $F_{-}, r_{-}$, and $t$-values reported in the results sections, we classified a specific task as demonstrating a positive effect of interpreting if the $p$-value was below .05 , and if the direction of the effect was in favour of the group of interpreters as compared to the controls, or in favour of interpreters with more experience or training. A specific task was classified as showing no effect if the $p$-value was above .05. None of the tasks showed a significantly negative effect of interpreting, interpreter experience, or interpreter training.

\section{Response-Distractor Inhibition}

For more detailed information on the tasks included as measures of the ResponseDistractor Inhibition component, see Table 3.

Regarding the first research question (On which executive process do interpreters score better than controls?), we determined that eight studies have tested the interpreter advantage in Response-Distractor Inhibition using 10 tasks in a cross-sectional design with between-group comparison including at least one group of interpreters and at least one group of non-interpreters. From these, three tasks (30\%) show better performance for groups of interpreters and seven tasks (70\%) show no effect of interpreting groups (either professionals, novices or students).

Regarding the second research question (Which executive process is related to interpreting experience?), we found that two studies have tested this in Response-Distractor 
Inhibition using three tasks. One study, reporting on one task, has used a cross-sectional design with between-group comparisons on professional interpreters with different levels of experience and the other study has used a cross-sectional design with a correlational analysis. On one out of three tasks (33\%), an advantage was found for interpreters with more experience, on the other two tasks (67\%), no advantage was found.

Regarding the third research question (Which executive process shows most progress during interpreter training?), we determined that two studies have used a cross-sectional design with between-group comparisons on two or more groups of interpreter trainees and one study has used a longitudinal design. None of these studies $(0 \%)$ show a relationship between interpreter training and Response-Distractor Inhibition.

For a graphical summary of the results on Response-Distractor Inhibition, see Figure 2. 
Table 3. Information about Response-Distractor Inhibition tasks included in systematic review. Cross-sect. $=$ study with a cross-sectional design. 1 group $\mathrm{I}=$ only one group of interpreters was included. $>1$ group IE $=$ more than one group of interpreters was included based on their experience. $>1$ group IT = more than one group of interpreters was included based on the length of training. Corr. = correlational. I+ = interpreter advantage. IE $+=$ advantage for interpreters with more experience. IE- = advantage for interpreters with less experience. $n s .=$ no significant difference or correlation .

\begin{tabular}{llll}
\hline \hline Article & Research design & Task(s) & Results \\
\hline Babcock et al. (in press) & Cross-sect., 1 group I. & Stroop & $n s$. \\
& Cross-sect., 1 group I. & ANT & $n s$. \\
\hline Babcock et al. (2015) & Cross-sect., 1 group I. & Task-switching & $n s$. \\
& Cross-sect., > 1 group IT. & Task-switching & $n s$. \\
\hline Dong et al. (2014) & Cross-sect., 1 group I. & Flanker & $n s$. \\
& Cross-sect., >1 group IT. & Flanker & $n s$. \\
\hline Dong et al. (2016) & Cross-sect., 1 group I. & Stroop & $n s$. \\
& Longitudinal, IT & Stroop & $n s$. \\
\hline Kopke et al. (2006) & Cross-sect., 1 group I. & Stroop & I+ \\
& Cross-sect., 1 group IE. & Stroop & IE- \\
\hline Morales et al (2015) & Cross-sect., 1 group I. & ANT & $n s$. \\
\hline Timarová et al. (2014) & Cross-sect., corr. IE. & Anti-saccade & $n s$. \\
& Cross-sect., corr. IE. & Flanker & IE+ \\
\hline Woumans et al. (2015) & Cross-sect., 1 group I. & Simon & I+ \\
& Cross-sect., 1 group I. & ANT & I+ \\
\hline Yudes et al. (2011) & Cross-sect., 1 group I. & Simon & $n s$. \\
\hline
\end{tabular}




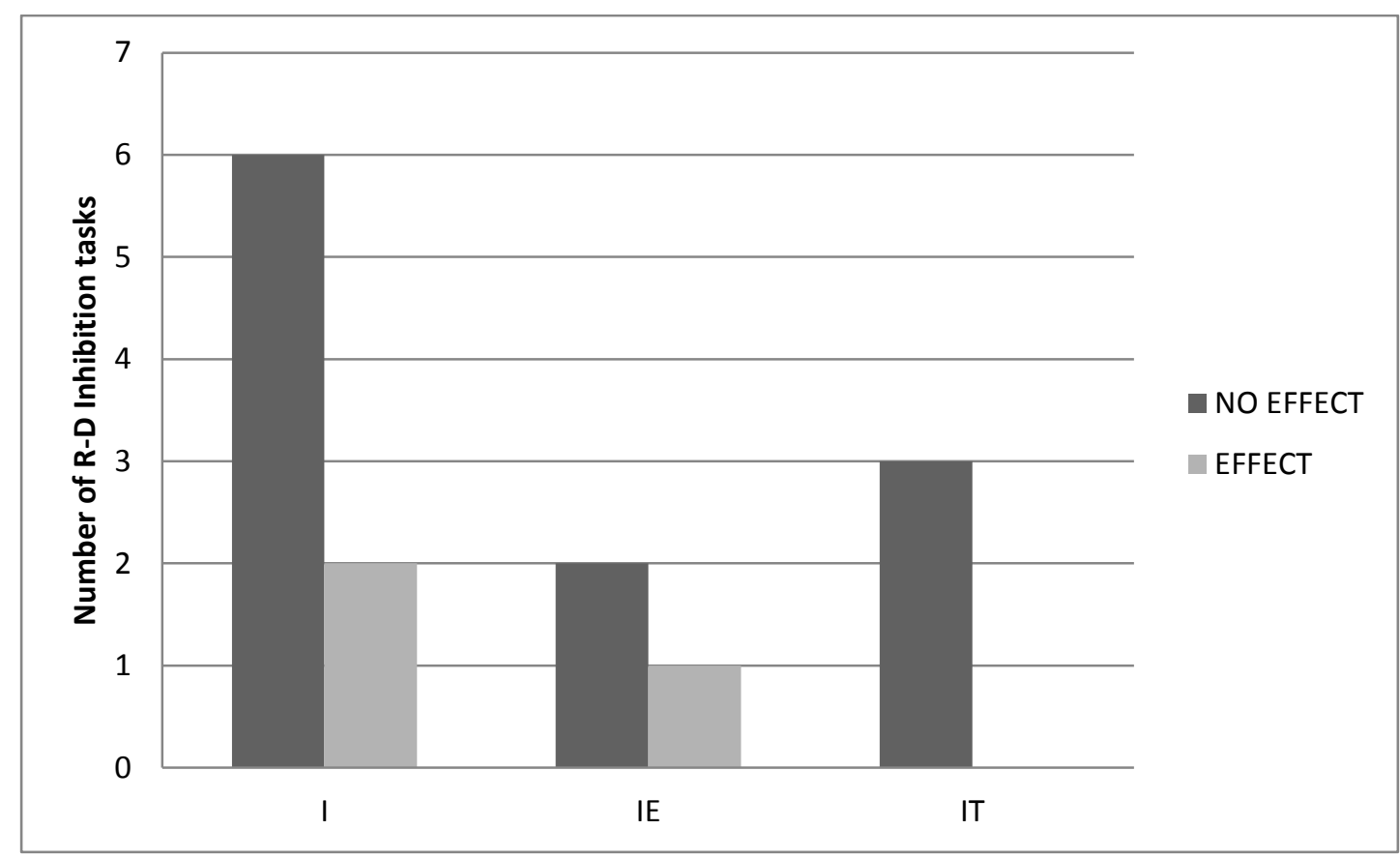

Figure 2. The results of studies on the interpreting advantage in Response-Distractor Inhibition according to the study design and the research question being answered. $\mathrm{I}=$ design with between-group comparison between interpreters and controls. IE = cross-sectional design with between-group comparison between interpreters with differing levels of experience or with correlational analyses. IT = cross-sectional design with between-group comparison between interpreter trainees with differing levels of training or longitudinal design.

\section{Shifting}

For more detailed information on the tasks included as measures of the Shifting component, see Table 4.

Regarding the first research question (On which executive process do interpreters score better than controls?), we discerned four studies that tested the interpreter advantage in Shifting using a cross-sectional design with between-group comparisons including at least one group of interpreters. From these four studies, all of them (100\%) found a positive effect of interpreting groups (either professionals, novice, and students) compare to non-interpreting group (either bilinguals, multilinguals, monolinguals, or language teachers). 
Table 4. Information about Shifting tasks included in systematic review. Cross-sect. = study with a cross-sectional design. 1 group $\mathrm{I}=$ only one group of interpreters was included. $>1$ group IE $=$ more than one group of interpreters was included based on their experience. $>1$ group IT = more than one group of interpreters was included based on the length of training. Corr. $=$ correlational. $\mathrm{I}+=$ interpreter advantage. $\mathrm{IE}+=$ advantage for interpreters with more experience. $\mathrm{IT}+=$ advantage for interpreters with more training. ns. $=$ no significant difference or correlation.

\begin{tabular}{llll}
\hline \hline Article & Research design & Task(s) & Results \\
\hline Babcock et al. (in press) & Cross-sect., 1 group I. & Task-switching & I+ \\
\hline Dong et al. (2014) & Cross-sect., >1 group I. & WCST & I+ \\
& Cross-sect., >1 group IT. & WCST & IT+ \\
\hline Dong et al. (2016) & Cross-sect., 1 group I. & Task-switching & I+ \\
& Longitudinal, IT. & Task-switching & IT+ \\
\hline Macnamara et al. (2014) & Longitudinal, IT. & WCST & IT+ \\
\hline Macnamara et al. (in press) & Longitudinal, IT. & WCST & IT+ \\
\hline Timarová et al. (2014) & Cross-sect., corr. IE. & Number-letter & $n s$. \\
\hline Yudes et al. (2011) & Cross-sect., 1 group I. & WCST & I+ \\
\hline \hline
\end{tabular}

Regarding the second research question (Which executive process is related to interpreting experience?) we established that one study used a cross-sectional design with correlational analyses and this study reports no correlation $(0 \%)$ between interpreting expertise and shifting ability.

Regarding the third research question (Which executive process is related to experience in interpreting?), we found three studies employing a longitudinal design and one study involving a cross-sectional design with between-group comparisons on two or more groups of interpreter trainees. These four studies all show better performance for students with longer interpreting training $(100 \%)$.

For a graphical summary of the results on Shifting, see Figure 3. 


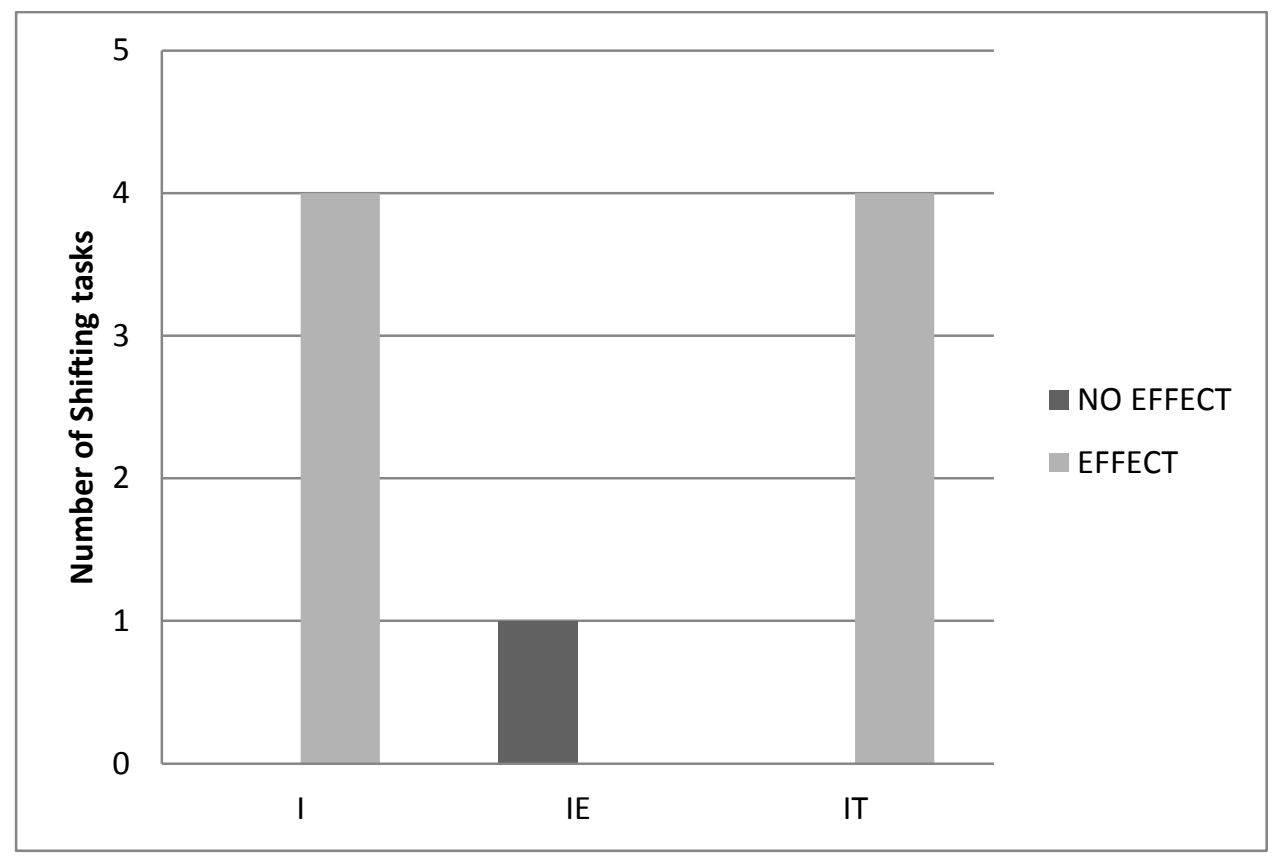

Figure 3. The results of studies on the interpreting advantage in Shifting according to the study design and the research question being answered. I = design with between-group comparison between interpreters and controls. IE = cross-sectional design with betweengroup comparison between interpreters with differing levels of experience or with correlational analyses. IT = cross-sectional design with between-group comparison between interpreter trainees with differing levels of training or longitudinal design.

\section{Updating}

For more detailed information on the tasks included as measures of the Updating component, see Table 5. 
Table 5. Information about Updating tasks included in systematic review. Cross-sect. = study with a cross-sectional design. 1 group I = only one group of interpreters was included. $>1$ group IE $=$ more than one group of interpreters was included based on their experience. $>1$ group IT = more than one group of interpreters was included based on the length of training. $\mathrm{I}+=$ interpreter advantage. $\mathrm{IE}+=$ advantage for interpreters with more experience. $\mathrm{ns} .=$ no significant difference or correlation.

\begin{tabular}{|c|c|c|c|}
\hline Article & Research design & Task & Results \\
\hline Babcock et al. (in press) & Cross-sect., 1 group of I. & Complex span & $\mathrm{I}+$ \\
\hline Christoffels et al. (2006) & Cross-sect., 1 group of I. & Complex span & $\mathrm{I}+$ \\
\hline \multirow[t]{2}{*}{ Dong et al. (2016) } & Cross-sect., 1 group of I. & N-back task & $\mathrm{I}+$ \\
\hline & Longitudinal, IT. & N-back task & $\mathrm{IT}+$ \\
\hline Injoque-Ricle (2015) & Cross-sect., corr. IE. & Complex span & ns. \\
\hline \multirow[t]{2}{*}{ Köpke et al. (2006) } & Cross-sect., 1 group I. & Complex span & $\mathrm{I}+$ \\
\hline & Cross-sect., $>1$ group IE. & Complex span & ns. \\
\hline \multirow[t]{2}{*}{ Liu et al. (2004) } & Cross-sect., >1 group IE. & Complex span & ns. \\
\hline & Cross-sect., $>1$ group IT & Complex span & ns. \\
\hline Macnamara et al. (2014) & Longitudinal, IT. & Complex span (2) & ns. \\
\hline Macnamara et al. (in press) & Longitudinal, IT. & Complex span & ns. \\
\hline Morales et al. (2015) & Cross-sect., 1 group I. & $\mathrm{N}$-back task & $\mathrm{I}+$ \\
\hline \multirow[t]{2}{*}{ Signorelli et al. (2011) } & Cross-sect., 1 group I. & Complex span & $\mathrm{I}+$ \\
\hline & Cross-sect., $>1$ group IE. & Complex span & ns. \\
\hline Stavrakaki et al. (2012) & Cross-sect., 1 group of I. & Complex span & ns. \\
\hline Timarová et al. (2014) & Cross-sect., corr. IE & $\mathrm{N}$-back task & ns. \\
\hline \multirow[t]{2}{*}{ Tzou et al. (2011) } & Cross-sect., 1 group I. & Complex span & $\mathrm{I}+$ \\
\hline & Cross-sect., $>1$ group IT. & Complex span & ns. \\
\hline Yudes et al. (2011) & Cross-sect., 1 group I. & Complex span & I+ \\
\hline
\end{tabular}

Regarding the first research question (On which executive process do interpreters score better than controls?) we determined nine tasks that have tested the interpreter advantage in Updating using a cross-sectional design with between-group comparisons. From these nine tasks, eight updating tasks ( $89 \%$ ) found a positive effect for one group of interpreters (either professionals or trainees) as compared to a non-interpreting control group 
(either bilinguals, multilinguals, monolinguals, or language teachers) and one task found no effect $(11 \%)$.

Regarding the second research question (Which executive process is related to interpreting experience?), we found five studies that have used a cross-sectional design with correlational analyses $(N=2)$ or a between-group comparisons that involve more than one group of interpreters $(N=3)$. Of these five studies, none show any correlation between interpreting experience and updating skill; neither do they reveal a group difference in updating when comparing more experienced interpreters with interpreter trainees $(0 \%)$.

Regarding the third research question (Which executive process shows most progress during interpreter training?), we discerned five studies which have used a longitudinal design $(N=3)$ or a cross-sectional design with between-group comparisons on two or more groups of interpreter trainees $(N=2)$. Only one longitudinal study $(20 \%)$ shows a positive effect of interpreting training on Updating. The four other studies (80\%) show no relation between interpreting training and Updating skill; neither do they reveal any group differences when comparing interpreter trainees in their different stage of training.

For a graphical summary of the results on Updating, see Figure 4. 


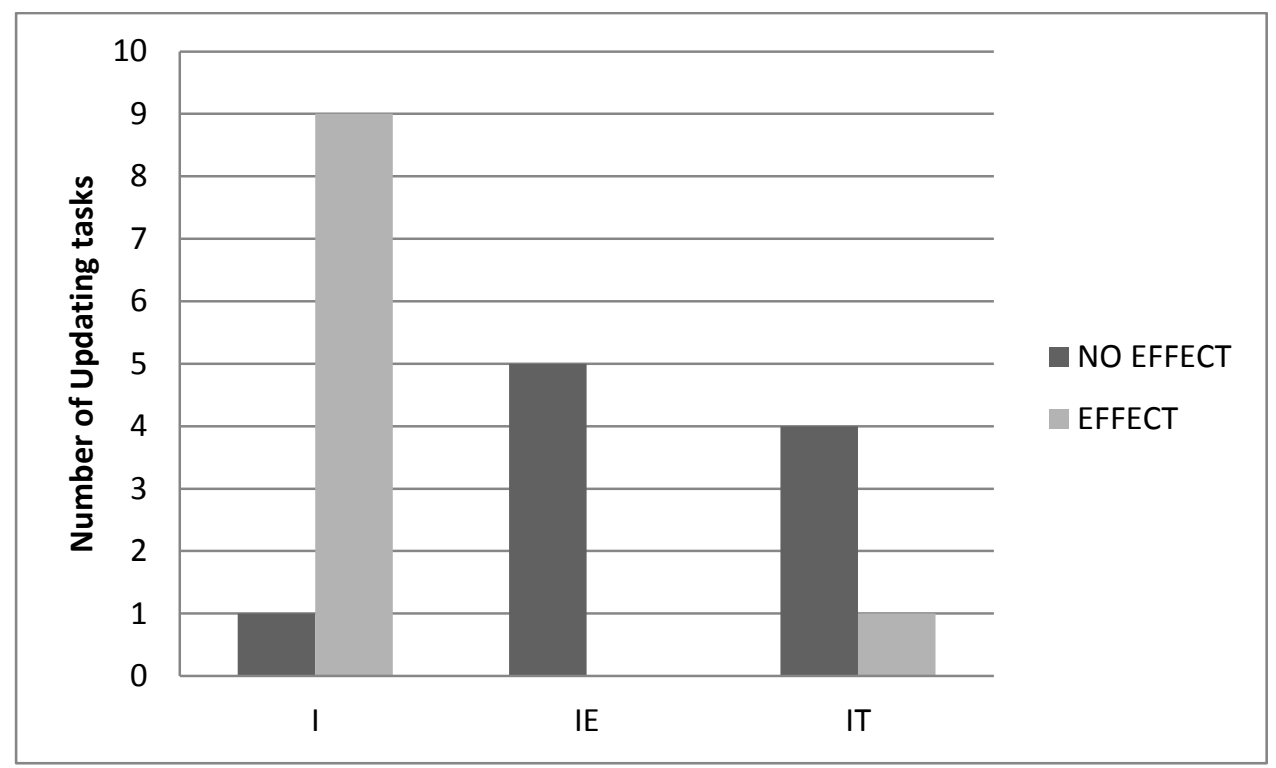

Figure 4. The results of studies on the interpreting advantage in Updating according to the study design and the research question being answered. I = design with between-group comparison between interpreters and controls. IE = cross-sectional design with betweengroup comparison between interpreters with differing levels of experience or with correlational analyses. IT = cross-sectional design with between-group comparison between interpreter trainees with differing levels of training or longitudinal design.

\section{Discussion}

The goal of this systematic review was to clarify and classify the relation between components of executive function and interpreting to see where and when we can find any advantage for this bilingual group. To this aim we have answered to three research questions on the relationship between interpreter experience and training and different components of executive functioning by conducting a systematic review of the available literature on this topic. A total of 17 articles were found and the tasks included in these articles were classified according to the empirically validated 'unity and diversity model' (Friedman \& Miyake, 2004; Miyake et al., 2000) into the following three components: Response-Distractor Inhibition, Shifting, and Updating. The results have revealed that interpreting experience and interpreter training differently affect each of these three components. The following section is structured according to the three research questions that were outlined at the end of the introduction. 


\section{On which executive process do interpreters score better than controls?}

Our analysis on between-group comparisons with at least one group of interpreters and at least one group of controls has revealed interpreter advantages on two of the three executive processes: Shifting (e.g. Babcock \& Vallesi, 2015; Dong \& Liu, 2016; Yudes et al., 2011), and Updating (e.g. Babcock \& Vallesi, 2015; Christoffels et al., 2006; Köpke \& Nespoulous, 2006); but not on Response-Distractor Inhibition (Dong \& Liu, 2016; Köpke \& Nespoulous, 2006; Morales et al., 2015). On Shifting, this advantage could be found in all incorporated studies; on Updating, only one study out of nine reported no significant differences between interpreters and controls (Stavrakaki, Megari, Kosmidis, Apostolidou, \& Takou, 2012). In contrast, a majority of studies found no differences on tasks tapping into the Response-Distractor Inhibition component of executive functioning (Babcock \& Vallesi, 2015; Köpke \& Nespoulous, 2006; Morales et al., 2015). Despite this general trend, two studies reported an interpreter advantage on Response-Distractor Inhibition (Köpke \& Nespoulous, 2006; Woumans et al., 2015). In both occasions, the contradictory finding of an inhibitory advantage for interpreters can be related to the selection of the control groups. Woumans and colleagues (2015) only found an advantage for interpreters when their performance was contrasted to control groups of monolinguals and unbalanced bilinguals but not to a control group of balanced bilinguals. In the study by Köpke and Nespoulos (2006), an interpreter advantage on Response-Distractor Inhibition was selectively found for novice interpreters but not for expert interpreters when their performance was compared to a bilingual control group. Moreover, the novice interpreters outperformed the two other groups only on the L1 but not on the L2 condition of a bilingual Stroop task (Stroop, 1935). This finding was assumed to result from a lack of balance between the languages in the novice interpreters, the dominant language being less affected by interference from the non-dominant language than vice versa (Köpke \& Nespoulous, 2006).

Interestingly, interpreter advantages on Shifting and Updating have been observed over groups of bilingual and monolingual controls, alike. This is remarkable because previous studies have suggested the existence of bilingual advantages on these two components of executive functioning (e.g. Bialystok, 2010; Prior \& MacWhinney, 2010; but also see Rosselli, Ardila, Lalwani, \& Velez-Uribe, 2016). The acquisition of interpreting skills, thus, seems to rely to a larger extent on executive processes than bilingualism in general. This additional effect can be understood as an illustration of interpreting as extreme language control (Hervais-Adelman, Moser-Mercer, Michel, et al., 2015). Positioned at the extreme of 
a continuum ranging from bilingual language activities that require only little control (e.g. communication in only one language) to activities that require extensive control (e.g. simultaneous activation of two languages), interpreting is related to more domain-general control abilities in Shifting and Updating than bilingualism in general. Even though bilingual advantages have also been reported on Response-Distractor Inhibition (e.g. Blumenfeld \& Marian, 2014; Kazemeini \& Fadardi, 2016; but also see Morton \& Harper, 2007), our review suggests that interpreting does not entail additional requirements related to this skill.

\section{Which executive process is related to interpreting experience?}

Our analysis of between-group comparisons with more than one group of interpreters based on their years of experience and of correlational studies has revealed no relationship whatsoever between interpreter experience and Updating (Injoque-Ricle et al., 2015; Liu et al., 2004; Timarova et al., 2014) or Shifting (Timarova et al., 2014), but mixed results regarding this relationship on Response-Distractor Inhibition (Köpke \& Nespoulous, 2006; Timarova et al., 2014). Interestingly, the tasks used to measure Response-Distractor Inhibition show different sensitivity: on a Stroop task, Köpke and Nespoulos (2006) found no difference between professional interpreters and interpreter students on a Stroop task. Timarová and colleagues (2014) used two different inhibition tasks with mixed results: a significant correlation between Response-Distractor Inhibition and interpreter experience was only found on the flanker task, but not on the anti-saccade task. It should be noted that only the antisaccade task was included in the initial version of the 'unity and diversity' model as a valid measure of the latent variable 'Inhibition' (Miyake et al., 2000) In this systematic review, we chose to include measures of Resistance to Distractor Interference, such as the flanker, as examples of an overarching Response-Distractor Inhibition component, because previous research has shown that these measures are closely related to tasks of Response Inhibition, such as the anti-saccade and the Stroop task (Friedman \& Miyake, 2004). The outcome of our review suggest that both inhibitory subcomponents may be differently sensitive to the effects of interpreter experience with a selective effect only on Resistance to Distractor Interference but not on Response Inhibition (Timarova et al., 2014)

The combination of interpreter advantages on Shifting and Updating when compared to controls with the absence of a relationship between these skills and amount of interpreter experience, seems to suggest that training effects related to daily interpreting practice are limited in scope. One possible explanation for this surprising finding is that professional 
interpreters have reached such a high level of expertise that the processes involved in interpreting have become automatised and no longer require the recruitment of domaingeneral cognitive control in their daily professional practice (Hervais-Adelman, MoserMercer, \& Golestani, 2015).

\section{Which executive process shows most progress during interpreter training?}

In contrast to the previous questions, our analysis of longitudinal studies on the effect of interpreter training has revealed a consistent pattern of results with no exceptions. While all reviewed studies show a training effect on Shifting (Dong \& Liu, 2016; Macnamara \& Conway, 2014; Macnamara \& Conway, in pressin press), only one of the reviewed studies shows a positive effect of interpreting training on updating (Dong \& Liu, 2016) and not a single study reports any correlation between interpreting training and Response-Distractor Inhibition (Dong \& Liu, 2016; Dong \& Xie, 2014).

In fact, Shifting was the only cognitive control component that could be significantly improved by interpreting training as compared to other types of training such as second language acquisition (Dong \& Xie, 2014). Possibly, this training-related increase in Shifting skills as shown by behavioural measurements is related to the training-related decline in neural activity of the caudate nucleus as revealed by a recent longitudinal study on the effect of interpreter training on brain functions (Hervais-Adelman, Moser-Mercer, \& Golestani, 2015). The authors of that neuroimaging study do not specify exactly which of the three components of executive functioning would be affected most by simultaneous interpreting; however, our systematic review suggests a specific role for the Shifting component.

One longitudinal study shows a significant effect of interpreting training on Updating skills (Dong \& Liu, 2016), but this improvement is not specific to interpreters students since the control group of translators display similar progress. In other words, apart from general training effects not specifically related to interpreting, interpreters tend to have the same level of updating skill regardless of their level of training and experience. The absence of a direct relation between interpreting performance and Updating in correlational studies seems to suggest a limited and short-term trainability of this executive process in relation to intense language training in translation or interpreting. To a certain extent, thus, the interpreter advantage on Updating is independent of experiential factors such as training and experience, which could be explained as an example of auto-selection: people who choose to become interpreters have better updating skills, even at the onset of their interpreter training. Although 
there is no evidence supporting any change in Updating by interpreting training in longitudinal studies, this skill is still reported as a crucial predictor for interpreting performance both at the beginning and at the end of students' training indicating that the students with better Updating skills perform better on an interpreting task (Cai et al., 2015; Macnamara \& Conway, in pressin press; Tzou et al., 2012). Previous research has already shown that self-selected interpreting students have higher Shifting abilities and are less negatively affected by anxiety than controls (Timarova $\&$ Salaets, 2011). The data from this systematic review suggest that, in addition to Shifting and anxiety, Updating abilities form a third domain on which interpreting students differ from controls. We therefore recommend future research on the interaction between interpreting and executive functioning to take these differences into account as they probably reflect pre-existing individual differences between interpreters and non-interpreters that are not specifically related to interpreter expertise or training.

\section{Integration into theory of executive functions}

While answering the three research questions above, it became clear that some executive function components, such as Shifting, are more sensitive to interpreting experience and training, and others, such as Updating, are not. The two factors of interpreting training and experience can be considered as environmental influences, which are the influences that give rise to behavioural differences in individuals who are exposed to different environments. In this case the Shifting component is sensitive to enviromental influences, while Updating is not. In contrast, the Response-Distractor Inhibtion factor is neither sensitive to interpreting training nor experience, but rather to the bilingualism factor in general, which indicates that this component is less sensitive to environmental influences than Shifting but more than Updating. The general interaction of different variables and executive function components based on the systematic review can be seen in Figure 5. 


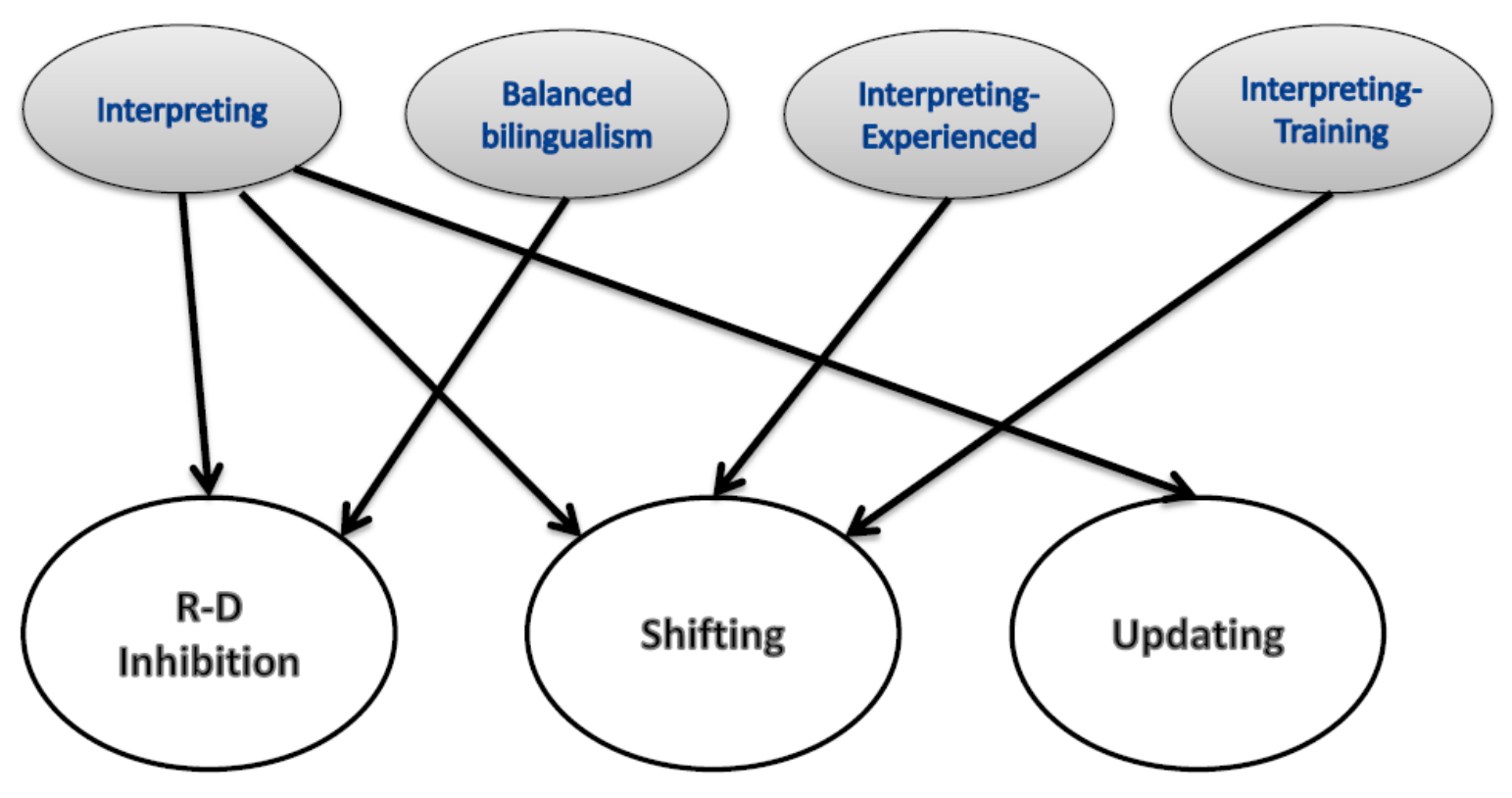

Figure 5. Interaction of reviewed variables, balanced bilingualism and EF components.

This pattern of selective trainability of executive functions may be integrated into the ACE (A: additive genetic, C: common environmental, E: non-shared environmental) genetic model of Friedman and colleagues (2016), who have examined genetic and environmental stability and change in three executive processes, measured with latent variables, from late adolescence to early adulthood in twins. The study reports that individual differences in executive functions are relatively heritable and stable by late adolescence, but are still sensitive to environmental influences with different degrees of sensitivity for each of its components.

The ACE genetic model has revealed that this sensitivity is lowest for the Updatingspecific component with little or no room for changes in function of environmental influences. In line with this, our systematic review reports that the Updating skill is not sensitive to interpreting training and interpreting experience. As for Response-Distractor Inhibition, the ACE model shows that nearly $20 \%$ of its variability is sensitive to environmental factors. Our review also shows a limited and selective trainability of Inhibition as a function of bilingualism in general but not of interpreting specifically. Highest sensitivity to environmental factors is found on Shifting, with over $20 \%$ of its variability attributable to non-genetic factors. In our review, Shifting likewise turns out to be the only executive process that is sensitive to interpreter training. 
To conclude, our systematic review shows that all executive functions are better in interpreting groups compared to non-interpreters, which strongly suggests the role of genetic heritability and auto-selection of interpreting trainees. However, different components show different sensitivity to environmental influences, which the highest is for shifting and the lowest for updating. Considering these finding we should be careful about the interpretation of these results as mentioned in Friedman and colleagues (2016: 17): “... heritability should not be interpreted as an index of the extent to which EFs are amenable to environmental interventions. In particular, high heritability of EFs should not be used as a basis for pessimism about the potential for environmental interventions and training."

While interpreting the results of this systematic review, we must take into account a number of limitations we had to face in the process of this study. The first one is the difference in the number of tasks across the three components of executive functioning. Nearly half of the reviewed studies reported on Updating tasks; in contrast, we could only find eight Shifting tasks. Therefore, we recommend further studies to include tasks that measure the three components within the same study (e.g. Dong \& Liu, 2016). Related to this, a second limitation of our review is that our answers to the second and third research questions for Shifting and Response-Distractor Inhibition could only be based on two studies and should, therefore, be interpreted with caution. A third limitation of this review is the small variability in research designs that have been used to test the interpreter advantage. While over two out of three studies have chosen for a cross-sectional design with between-group comparisons, only three studies have used a longitudinal design and only two studies report having conducted correlational analyses. On Response-Distractor Inhibition, we could not find a single longitudinal study to test the trainability of this skill in interpreters. Therefore, we strongly recommend further investigation into the effect of interpreter training using a prepost-test or a correlational design as these designs have most potential to single out effects that are specifically related to interpreting experience and training from effects related to genetic or other non-environmental factors. 


\section{Conclusion}

This systematic review has demonstrated the added value of the 'unity and diversity' model of executive functions for answering research questions on the locus of the interpreter advantage. First, our review of cross-sectional research with between-group comparisons has clearly revealed that interpreter advantages in executive functioning are only found on Shifting and Updating, but not on Response-Distractor Inhibition. Second, our review of longitudinal designs has further restricted the effects of interpreter training to Shifting. We conclude that interpreting is a form of extreme language control, which taps into specific domain-general control requirements related to Shifting and Updating abilities. While interpreter trainees seem to possess superior Updating abilities at training onset, Shifting abilities may further develop during training. 


\section{References}

Arnett, P. A., Rao, S. M., Grafman, J., Bernardin, L., Luchetta, T., Binder, J. R., \& Lobeck, L. (1997). Executive functions in multiple sclerosis: An analysis of temporal ordering, semantic encoding, and planning abilities. Neuropsychology 11 (4), 535-544.

Babcock, L., \& Vallesi, A. (2015). Language control is not a one-size-fits-all languages process: evidence from simultaneous interpretation students and the $\mathrm{n}-2$ repetition cost. Frontiers in Psychology 6.

Babcock, L., \& Vallesi, A. (in press). Are simultaneous interpreters expert bilinguals, unique bilinguals, or both? Bilingualism: Language and Cognition.

Baddeley, A. (1992). Working memory. Science 255 (5044), 556-559.

Bayliss, D. M., Jarrold, C., Gunn, D. M., \& Baddeley, A. D. (2003). The complexities of complex span: Explaining individual differences in working memory in children and adults. Journal of Experimental Psychology-General 132 (1), 71-92.

Berg, E. A. (1948). A simple objective technique for measuring flexibility in thinking. Journal of General Psychology 39, 15-22.

Bialystok, E. (2010). Global-Local and Trail-Making Tasks by Monolingual and Bilingual Children: Beyond Inhibition. Developmental Psychology 46 (1), 93-105.

Blumenfeld, H. K., \& Marian, V. (2014). Cognitive control in bilinguals: Advantages in Stimulus-Stimulus inhibition. Bilingualism-Language and Cognition 17 (3), 610-629.

Cai, R. D., Dong, Y. P., Zhao, N., \& Lin, J. X. (2015). Factors contributing to individual differences in the development of consecutive interpreting competence for beginner student interpreters. Interpreter and Translator Trainer 9 (1), 104-120.

Christoffels, I. K., de Groot, A. M. B., \& Kroll, J. F. (2006). Memory and language skills in simultaneous interpreters: The role of expertise and language proficiency. Journal of Memory and Language 54 (3), 324-345.

Colom, R., Rebollo, I., Abad, F. J., \& Shih, P. C. (2006). Complex span tasks, simple span tasks, and cognitive abilities: A reanalysis of key studies. Memory \& Cognition 34 (1), 158-171.

Diamond, A. (2013). Executive Functions. Annual Review of Psychology 64, 135-168.

Dong, Y. P., \& Liu, Y. H. (2016). Classes in Translating and Interpreting Produce Differential Gains in Switching and Updating. Frontiers in Psychology 7. 
Dong, Y. P., \& Xie, Z. L. (2014). Contributions of second language proficiency and interpreting experience to cognitive control differences among young adult bilinguals. Journal of Cognitive Psychology 26 (5), 506-519.

Eriksen, B. A., \& Eriksen, C. W. (1974). Effects of noise letters upon identification of a target letter in a nonsearch task. Perception \& Psychophysics 16 (1), 143-149.

Fan, J., McCandliss, B. D., Sommer, T., Raz, A., \& Posner, M. I. (2002). Testing the efficiency and independence of attentional networks. Journal of Cognitive Neuroscience 14 (3), 340-347.

Friedman, N. P., \& Miyake, A. (2004). The relations among inhibition and interference control functions: A latent-variable analysis. Journal of Experimental PsychologyGeneral 133 (1), 101-135.

Friedman, N. P., Miyake, A., Altamirano, L. J., Corley, R. P., Young, S. E., Rhea, S. A., \& Hewitt, J. K. (2016). Stability and Change in Executive Function Abilities From Late Adolescence to Early Adulthood: A Longitudinal Twin Study. Developmental Psychology 52 (2), 326-340.

Garcia, A. M. (2014). The interpreter advantage hypothesis Preliminary data patterns and empirically motivated questions. Translation and Interpreting Studies 9 (2), 219-238.

Hallett, P. E. (1978). Primary and secondary saccades to goals defined by instructions. Vision Research 18 (10), 1279-1296.

Hernandez, A. E., Greene, M. R., Vaughn, K. A., Francis, D. J., \& Grigorenko, E. L. (2015). Beyond the bilingual advantage: The potential role of genes and environment on the development of cognitive control. Journal of Neurolinguistics 35, 109-119.

Hervais-Adelman, A., Moser-Mercer, B., \& Golestani, N. (2015). Brain functional plasticity associated with the emergence of expertise in extreme language control. Neuroimage 114, 264-274.

Hervais-Adelman, A., Moser-Mercer, B., Michel, C. M., \& Golestani, N. (2015). fMRI of Simultaneous Interpretation Reveals the Neural Basis of Extreme Language Control. Cerebral Cortex 25 (12), 4727-4739.

Hitch, G. J., \& Baddeley, A. D. (1976). Verbal reasoning and working memory. Quarterly Journal of Experimental Psychology 28, 603-621.

Injoque-Ricle, I., Barreyro, J. P., Formoso, J., \& Jaichenco, V. I. (2015). Expertise, Working Memory and Articulatory Suppression Effect: Their Relation with Simultaneous Interpreting Performance. Advances in Cognitive Psychology 11 (2), 56-63.

Jersild, A. T. (1927). Mental set and shift. Archives of Psychology, Whole No. 89. 
Jonides, J., \& Smith, E. E. (1997). The architecture of working memory. In M. D. Rugg (Ed.), Cognitive neuroscience. Cambridge, MA: MIT Press, 243-276.

Kazemeini, T., \& Fadardi, J. S. (2016). Executive Function: Comparing Bilingual and Monolingual Iranian University Students. Journal of Psycholinguistic Research 45 (6), 1315-1326.

Koch, I., Gade, M., \& Philipp, A. M. (2004). Inhibition of response mode in task switching. Experimental Psychology 51 (1), 52-58.

Koch, I., Gade, M., Schuch, S., \& Philipp, A. M. (2010). The role of inhibition in task switching: A review. Psychonomic Bulletin \& Review 17 (1), 1-14.

Köpke, B., \& Nespoulous, J.-L. (2006). Working memory performance in expert and novice interpreters. Interpreting 8 (1), 1-23.

Larson, G. E., Merritt, C. R., \& Williams, S. E. (1988). Information-processing and intelligence - Some implications of task complexity. Intelligence 12 (2), 131-147.

Liu, M., Schallert, D. L., \& Carroll, P. J. (2004). Working memory and expertise in simultaneous interpreting. Interpreting 6 (1), 19-42.

Logan, G. D. (1994). On the ability to inhibit thought and action: A user's guide to the stop signal paradigm. In D. Dagenbach \& T. H. Carr (Eds.), Inhibitory processes in attention, memory, and language. San Diego, CA: Academic Press, 189-239.

Macnamara, B. N., \& Conway, A. R. A. (2014). Novel evidence in support of the bilingual advantage: Influences of task demands and experience on cognitive control and working memory. Psychonomic Bulletin \& Review 21 (2), 520-525.

Macnamara, B. N., \& Conway, A. R. A. (in press). Working memory capacity as a predictor of simultaneous language interpreting performance. Journal of Applied Research in Memory and Cognition.

Macnamara, B. N., Moore, A. B., Kegl, J. A., \& Conway, A. R. A. (2011). Domain-general cognitive abilities and simultaneous interpreting skill. Interpreting 13 (1), 121-142.

Miyake, A., Friedman, N. P., Emerson, M. J., Witzki, A. H., Howerter, A., \& Wager, T. D. (2000). The unity and diversity of executive functions and their contributions to complex "frontal lobe" tasks: A latent variable analysis. Cognitive Psychology 41 (1), 49-100.

Monsell, S. (1996). Control of mental processes. In V. Bruce (Ed.), Unsolved mysteries of the mind: Tutorial essays in cognition. Hove, UK: Erlbaum, 93-148. 
Morales, J., Padilla, F., Gomez-Ariza, C. J., \& Bajo, M. T. (2015). Simultaneous interpretation selectively influences working memory and attentional networks. Acta Psychologica 155, 82-91.

Morris, N., \& Jones, D. M. (1990). Memory updating in working memory - The role of the central executive. British Journal of Psychology 81, 111-121.

Morton, J. B., \& Harper, S. N. (2007). What did Simon say? Revisiting the bilingual advantage. Developmental Science 10 (6), 719-726.

Navon, D. (1977). Forest before trees: The precedence of global features in visual perception. Cognitive Psychology 9 (3), 353-383.

Philipp, A. M., Gade, M., \& Koch, I. (2007). Inhibitory processes in language switching: Evidence from switching language-defined response sets. European Journal of Cognitive Psychology 19 (3), 395-416.

Philipp, A. M., \& Koch, I. (2009). Inhibition in Language Switching: What Is Inhibited When Switching Between Languages in Naming Tasks? Journal of Experimental Psychology-Learning Memory and Cognition 35 (5), 1187-1195.

Prior, A., \& MacWhinney, B. (2010). A bilingual advantage in task switching. BilingualismLanguage and Cognition 13 (2), 253-262.

Rogers, R. D., \& Monsell, S. (1995). Costs of a predictable switch between simple cognitive tasks. Journal of Experimental Psychology-General 124 (2), 207-231.

Rosselli, M., Ardila, A., Lalwani, L. N., \& Velez-Uribe, I. (2016). The effect of language proficiency on executive functions in balanced and unbalanced Spanish-English bilinguals. Bilingualism-Language and Cognition 19 (3), 489-503.

Scheil, J., \& Kleinsorge, T. (2014). N - 2 Repetition Costs Depend on Preparation in Trials n 1 and n - 2. Journal of Experimental Psychology-Learning Memory and Cognition 40 (3), 865-872.

Simon, J. R., \& Rudell, A. P. (1967). Auditory S-R compatibility - Effect of an irrelevant cue on information processing. Journal of Applied Psychology 51 (3), 300-\&.

Stavrakaki, S., Megari, K., Kosmidis, M. H., Apostolidou, M., \& Takou, E. (2012). Working memory and verbal fluency in simultaneous interpreters. Journal of Clinical and Experimental Neuropsychology 34 (6), 624-633.

Strobach, T., Becker, M., Schubert, T., \& Kuhn, S. (2015). Better dual-task processing in simultaneous interpreters. Frontiers in Psychology 6.

Stroop, J. R. (1935). Studies of interference in serial verbal reactions. Journal of Experimental Psychology 18, 643-662. 
Timarova, S., Cenkova, I., Meylaerts, R., Hertog, E., Szmalec, A., \& Duyck, W. (2014).

Simultaneous interpreting and working memory executive control. Interpreting 16 (2), 139-168.

Timarova, S., \& Salaets, H. (2011). Learning styles, motivation and cognitive flexibility in interpreter training Self-selection and aptitude. Interpreting 13 (1), 31-52.

Tzou, Y. Z., Eslami, Z. R., Chen, H. C., \& Vaid, J. (2012). Effect of language proficiency and degree of formal training in simultaneous interpreting on working memory and interpreting performance: Evidence from Mandarin-English speakers. International Journal of Bilingualism 16 (2), 213-227.

Unsworth, N., \& Engle, R. W. (2006). Simple and complex memory spans and their relation to fluid abilities: Evidence from list-length effects. Journal of Memory and Language 54 (1), 68-80.

Unsworth, N., \& Engle, R. W. (2007). On the division of short-term and working memory: An examination of simple and complex span and their relation to higher order abilities. Psychological Bulletin 133 (6), 1038-1066.

Van Dijk, R., Christoffels, I., Postma, A., \& Hermans, D. (2012). The relation between the working memory skills of sign language interpreters and the quality of their interpretations. Bilingualism-Language and Cognition 15 (2), 340-350.

Wang, J. H. (2016). The relationship between working memory capacity and simultaneous interpreting performance A mixed methods study on professional Auslan/English interpreters. Interpreting 18 (1), 1-33.

Woumans, E., Ceuleers, E., Van der Linden, L., Szmalec, A., \& Duyck, W. (2015). Verbal and Nonverbal Cognitive Control in Bilinguals and Interpreters. Journal of Experimental Psychology-Learning Memory and Cognition 41 (5), 1579-1586.

Yntema, D. B. (1963). Keeping track of several things at once. Human Factors 5 (1), 7-17.

Yudes, C., Macizo, P., \& Bajo, T. (2011). The influence of expertise in simultaneous interpreting on non-verbal executive processes. Frontiers in Psychology 2. 